\title{
A Method to Examine the Time-Space Relationship between Seismic Events
}

F.R.P. Basson, D.J.R.M. Ras Brentley, Lucas and Associates - Target Gold Mine, South Africa

Target Mine is a complex multiple stope environment with concentrated stoping operations in a relatively small area, at a depth of $2300 \mathrm{~m}$ below surface. The close proximity of working areas and geological structures require an understanding of the interactivity and correlation between seismic events to enable a continuous assessment of the state of stability and seismic damage potential on the mine. Three basic functionalities were identified as having potential to help in understanding the interactivity and correlation between seismic events; the visual playback of selected events in scaled time, visual correlation indicators between consecutive events, and the correlation frequency of events. Various scenarios were studied on Target (with in-house software) and results so far indicated that:

- Event clusters in space and time can be successfully identified.

- Bigger events appear to be mostly uncorrelated with small "telltale" events in the vicinity in time, but appear to be better correlated with other bigger events.

- When the cumulative number of correlations between bigger seismic events is plotted against time, risk profiles for specific areas or geological structures can be obtained.

\section{INTRODUCTION}

\subsection{Background on Target Gold Mine}

The Target gold prospect is a northwards continuation of the Welkom Goldfields in South Africa, which lies in the southernmost part of the Witwatersrand Basin and is located at Allanridge, $30 \mathrm{~km}$ north of the city of Welkom as depicted in Figure 1.

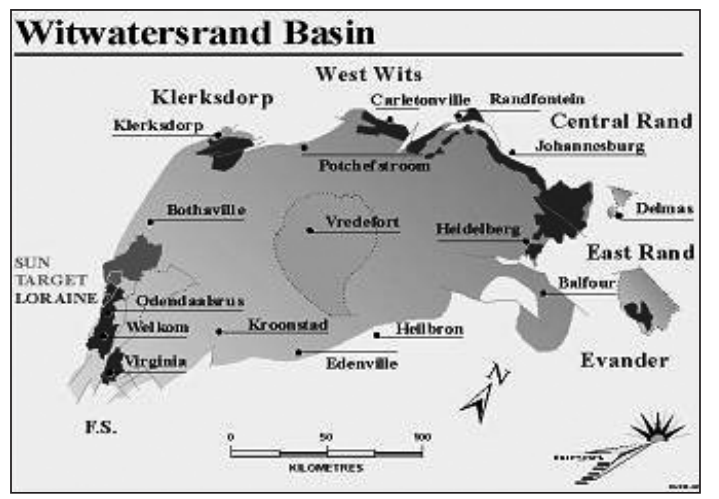

FIG. 1 Locality plan of Target in the Free State province of South Africa

The closely spaced reef occurrence allows massive mining methods with trackless equipment to be used. The application of these methods at a depth of $2300 \mathrm{~m}$ below surface requires de-stressing to be done to create a stress environment conducive to massive mining.

\subsection{Geology}

The Target orebody consists of sedimentary gold bearing reefs, situated mainly in a synclinal structure on the western limit of the Witwatersrand Basin. The syncline plunges to the north at $8^{\circ}$. Six reef packages have been identified as being of economic interest in the Target lease area, but currently only two (Elsburg and Dreyerskuil) are being exploited.

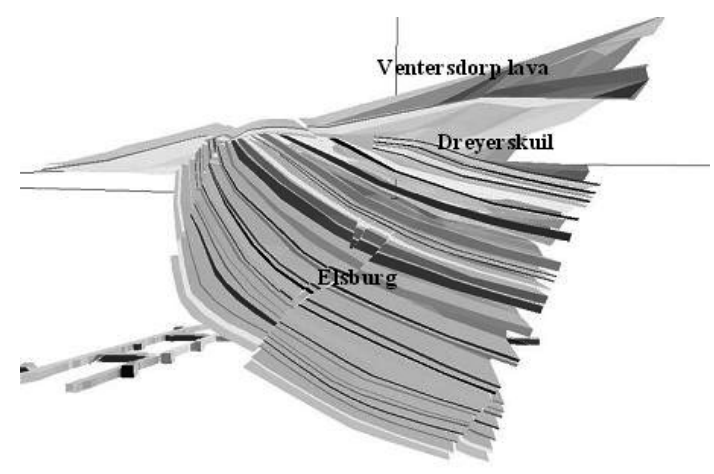

FIG. 2 A side view through a geological model of the Elsburg and Dreyerskuil reef horizons with only the gold bearing reefs indicated

The orebody is intersected by a number of faults and dykes.

\subsection{Geotechnical Setting}

The geotechnical challenge at Target is to maintain adequate stability and to prevent major damaging seismic events whilst mining large excavations in close proximity, at a depth of between $2200 \mathrm{~m}$ to $2500 \mathrm{~m}$ below surface. The host rock is generally very competent but tends to deform more elastically. It therefore has the capacity to store strain energy that is mainly released as seismic energy.

Massive mining is normally done at shallower depths where the virgin principal stress rarely exceeds $40 \mathrm{MPa}$, the correct sequencing of stopes and destressing of the massive stopes are thus of crucial importance. The Map3D boundary element code is mostly used for numerical modelling to guide decisions on this. 


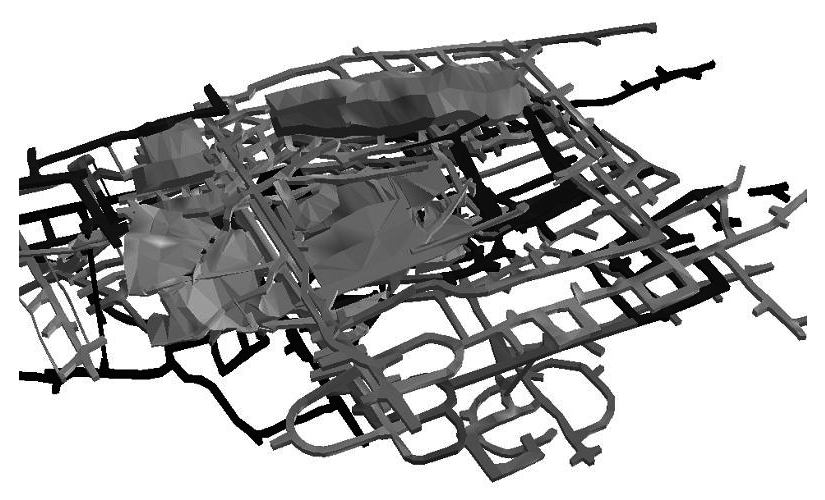

FIG. 3 A perspective view of Target Mine showing the complexity of the excavation configuration (the top tabular de-stress stope removed for clarity)

A seismic system consisting of accelerometers is installed underground to monitor the rock response to mining operations. This allows precautionary measures to be taken in an environment where the instability of a single excavation or movement on a geological structure can affect the entire operation. Apart from a tri-axial surface geophone, all the sensors underground are very close to the mining operation and can therefore be regarded as an exclusive near-field monitoring system.

The level of seismicity experienced at Target is indicated by the following graph.

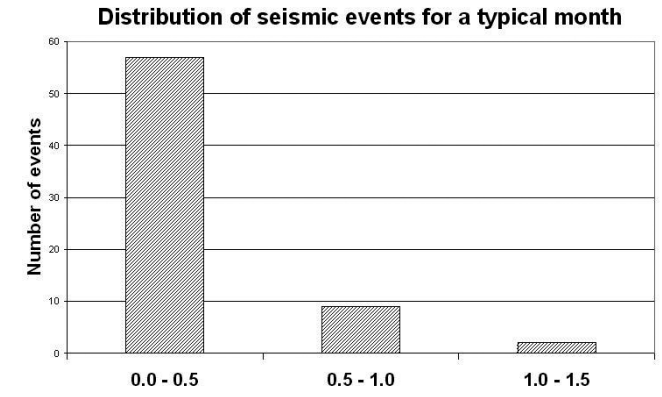

FIG. 4 Only events > local magnitude 0.0

\section{PURSUING A BETTER UNDERSTANDING}

It soon became clear that applying the normal methods for evaluating seismic data as done for other gold mines in South Africa (where relatively small volumes of rock are extracted over a large area, in essentially a planar configuration) may not be enough or fully applicable to Target.

To better interpret the recorded seismic emissions, it was decided to devise a method to study the space-time relationship of events. As prerequisites, the method had to be simple and robust and have the following functionalities:

- Visual playback of seismic events in scaled time.

- Visual correlation between seismic events according to selected criteria for space and time.

- Show the number of correlations obtained with the selected correlation parameters.

As no commercial software could be obtained that fulfill the requirements, a software package was developed in-house.

\section{SOFTWARE FUNCTIONALITY}

The developed software is a $2 \mathrm{D}$-solution that imports a bitmap-file and enables calibration of the picture for the correct plotting of the seismic events in space. Only one calibration is required as the information is saved and automatically obtained when the particular bitmap is opened in future.

Seismic information is imported and the information required is the event coordinates, date, time and magnitude. Selections of data can be made within the software and colour is used to distinguish between events that occur above, close to, or below the reef elevation.

The software provides the following output:

- Playback of the events in accelerated scaled time. This enables one to study the information as they occurred in time and space to try and establish trends.

- Correlation parameters can be set for time and space. When both these selected parameters are satisfied, a correlation is visually drawn on the picture. It was decided to ignore the $\mathrm{Z}$-values for the distance calculation, as it is well known to be inaccurate due to the distribution of the sensors on Target. Correlation in time only, can be obtained by providing an arbitrary large value for the distance correlation. An exceptionally large value for the distance correlation will always be satisfied, resulting in correlation in time only.

- Combining the drawing of correlation lines with the playback of events in scaled time, the formation of clusters can followed as they occur.

- The program gives the number of correlations that satisfied the selected parameters as well as the maximum number of correlations that could have been obtained. The significance of this functionality became apparent afterwards and will be explained in detail.

\section{RESULTS OBTAINED FROM TARGET INFORMATION}

\subsection{Clustering in Time and Space}

When varying the time and space filters, the associations between events quickly become apparent.

Closely spaced clusters can be successfully and quickly distinguished between, for further analysis if required.

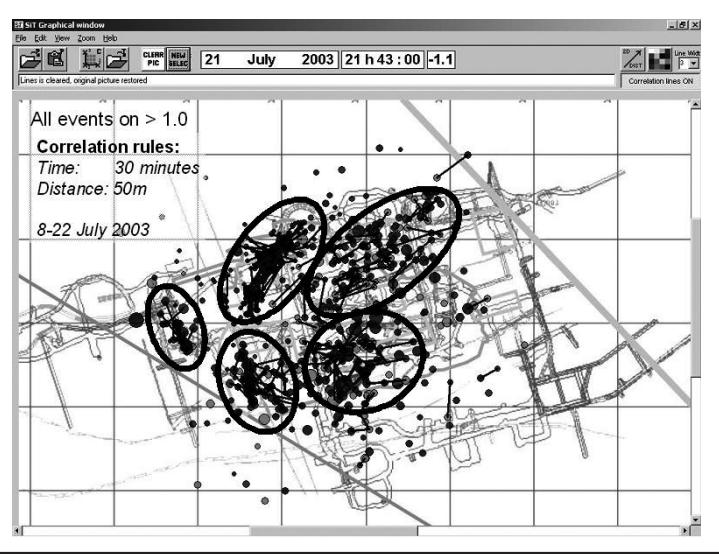

FIG. 5 Events that are related in time and space can be easily distinguished

The migration of event clusters on geological structures can also be easily followed as they develop in time.

\subsection{Correlation Between Smaller and Bigger Events}

Two schools of thought exist in seismology regarding the correlation between small and bigger events:

- The one thought is that small seismic events are precursors of rockmass instability that could lead 
to bigger seismic events. The small events could therefore be used to predict bigger events in time and space.

- The other school of thought maintains that the mechanisms of the very small and bigger events differ and that negates any potential in the prediction of bigger events from detailed knowledge of the very small events.

The correlation between small and bigger events was tested with the software on Target data and very few instances could be found were small events preceded bigger events. Bigger events appear mostly uncorrelated with the small events in the vicinity in time, but appear to be better correlated with other bigger events in the vicinity in time.

\subsection{Time Correlation Between Seismic Events}

The software was also used to establish how the rockmass normally responds in time after a bigger event did occur.

To obtain correlation in time only, an arbitrary large distance correlation-value was selected. If this distance-value is sufficiently large the condition will always be satisfied and the result is correlation dependant on time only.

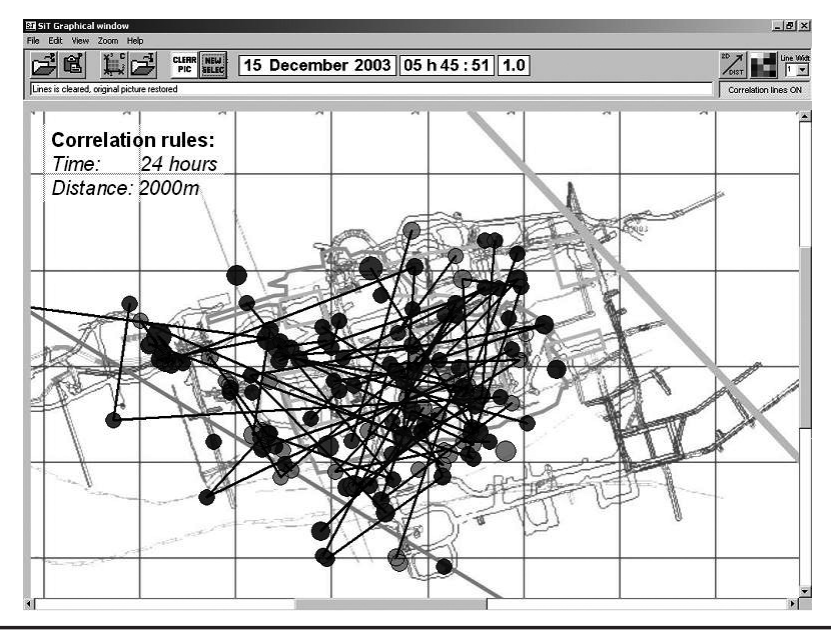

FIG. 6 Correlation in time only for bigger events recorded on Target for 2003

It is evident in Figure 6 that longer distance correlation between the bigger events was commonly observed. Short distance correlation $(<50 \mathrm{~m})$ between bigger seismic events was only recorded in one area close to a dyke.

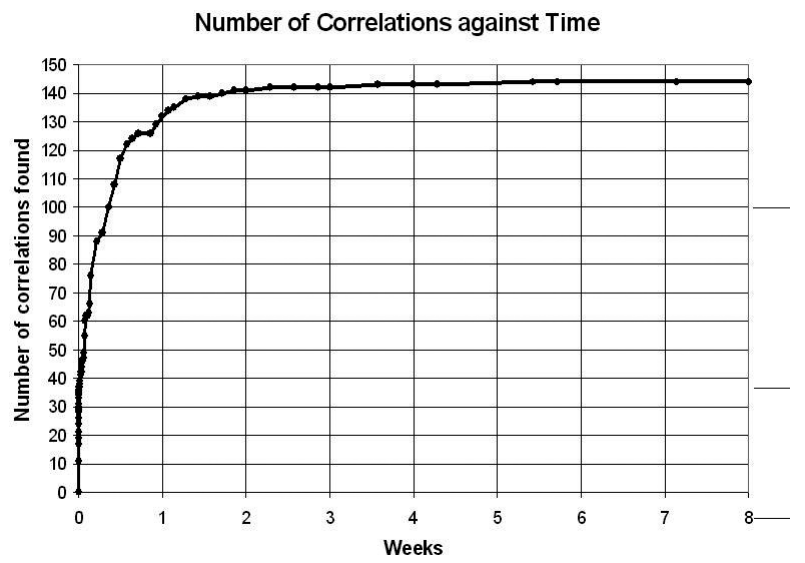

FIG. 7 The correlation between bigger events (greater than local magnitude 0.9) on Target for 2003
When plotting the cumulative number of correlated bigger events (after a bigger event occurred) against time, the gradient of the line is representative of the potential for a second bigger event to occur. A steep gradient represents a good chance for a correlated event, whereas a flatter gradient represents a lesser chance for a correlated second event. It is obvious from Fig.7 that there is initially a good change for a correlated second event to occur, but this probability then reduces with time to a background or nominal value after 3 to 4 weeks.

The graph can be divided into areas where the gradients (recurrence time for events) are roughly the same.

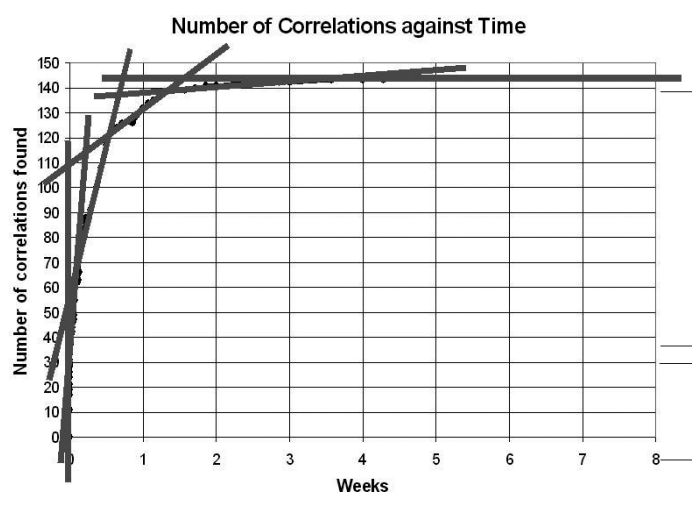

FIG. 8 Correlation in time only, divided into intervals of similar recurrence time for a second correlated seismic event

Figure 8 can then be manipulated to represent the probability for a second event to occur as indicated in Figure 9.

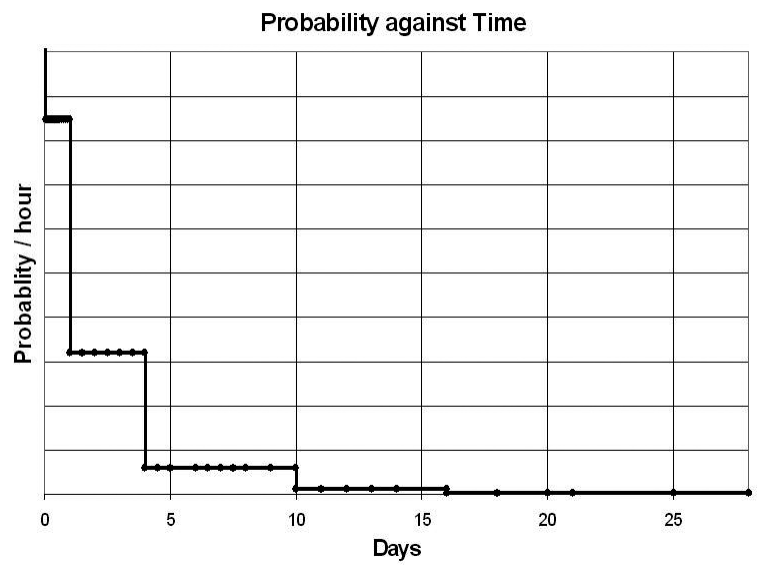

FIG. 9 The relative probability for a correlated second event from the time of the first bigger event

From Figure 9, it is obvious that the chance of having another big event occurring directly after a major event reduces substantially with time.

\subsection{Correlation Between Seismic Events in Time and Space}

From a safety decision-making point of view, the combined time and space relationship between bigger events can have a significant implication. A burning issue that often arises after a major damaging seismic event occurred is the time aspect; when is it safe to sent people in for either rescue operations or rehabilitation work? The biggest concern normally is the likelihood of reoccurrence of other events in the vicinity.

A correlation in time only is insufficient to evaluate this possibility, as the distance of the second event from the first 
must be taken into account. The required correlation is then done according to a selected time and distance. A decision is required beforehand on the perceived influence distance between events.

The gradient of the line obtained when plotting the number of correlations in space against time, represents the possibility of having a potentially damaging second event in the vicinity of the first event in time.

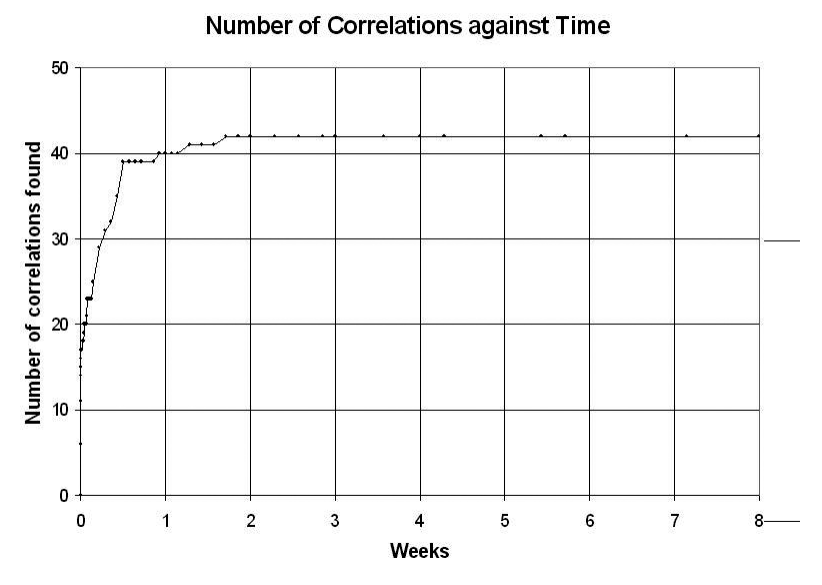

FIG. 10 Correlation in time and space for Target events that occurred within $80 \mathrm{~m}$ of each other

Four time periods with approximately constant recurrence times for bigger events can be distinguished from the obtained graph.

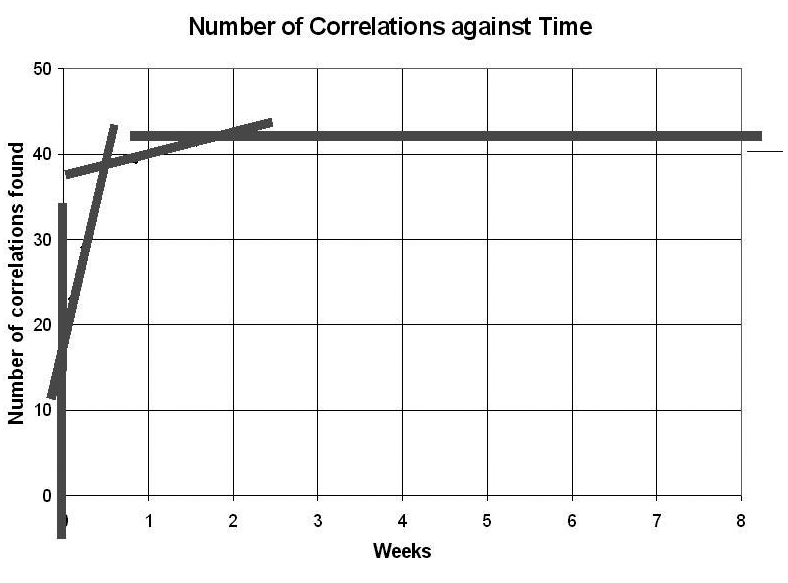

FIG. 11 Four clearly defined risk periods after the occurrence of a bigger event

The highest possibility for a correlated second bigger event is seconds after the first bigger event occurred. Thereafter the risk reduces and stays constant for 3 to 4 days. After 3 to 4 days the risk reduces substantially and after roughly 2 weeks the risk in the area returns to its nominal or background value with no correlation between events apparent.

\subsection{Characterisation of Geological Structures}

Correlation in time and space was also applied to different geological structures. The three selected structures intersected each other, resulting in a small portion of the events inevitably allocated to more than one structure. Even with this separation difficulty close to the intersection of the faults, the three selected structures gave distinct results. The selection of events associated with each structure was done in three dimensions, as the three structures dip at angles between $15^{\circ}$ and $31^{\circ}$ from vertical.

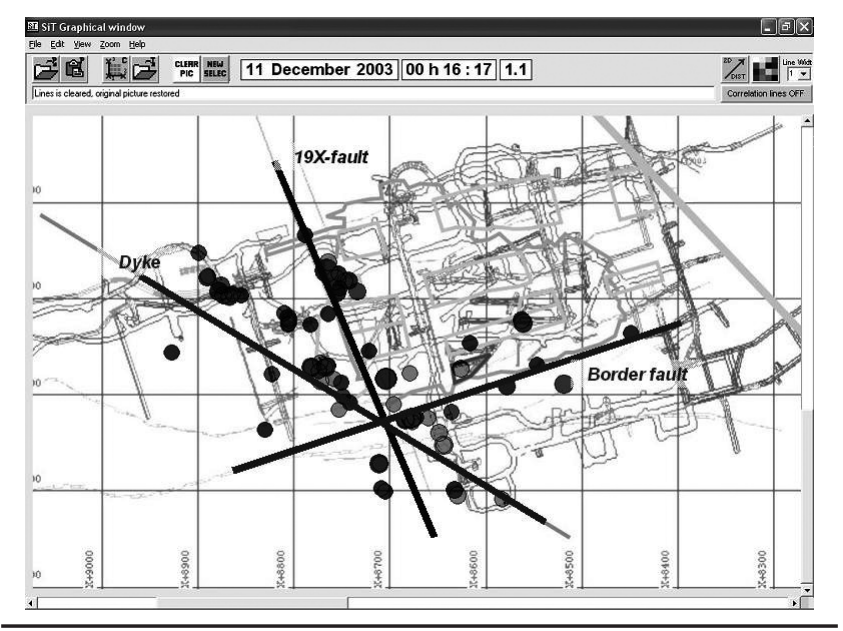

FIG. 12 Seismic events associated to the three selected discontinuities. This plan view can however be deceiving as all three the structures dips away from vertical with angles of between $15^{\circ}$ and $31^{\circ}$

A plot of the time-space relation for the three discontinuities gave a good indication of the behaviour of each structure after relatively large events occurred on the selected structures.

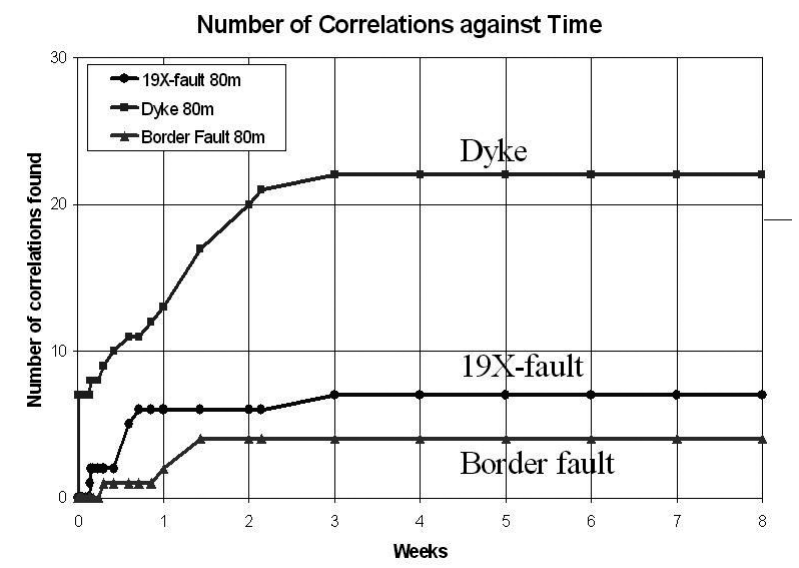

FIG. 13 Correlation in time (three selected discontinuities) for events that occurred within $80 \mathrm{~m}$ from the first large event

The three discontinuities have different risk profiles, with the dyke being the most hazardous structure of the three. It is also the only structure where associated seismic events could be expected to occur within seconds of the first event (in $30 \%$ of the incidents a second event within 1 second of the first). All structures show a better chance for a bigger seismic event to occur in the days directly after the first bigger event occurred. All were back at their background or nominal values after 2-3 weeks.

An interesting phenomenon that is apparent in the results of all three structures is the straight-line nature of the obtained lines. That indicates time periods where the risk of second associated events are approximately equal. 


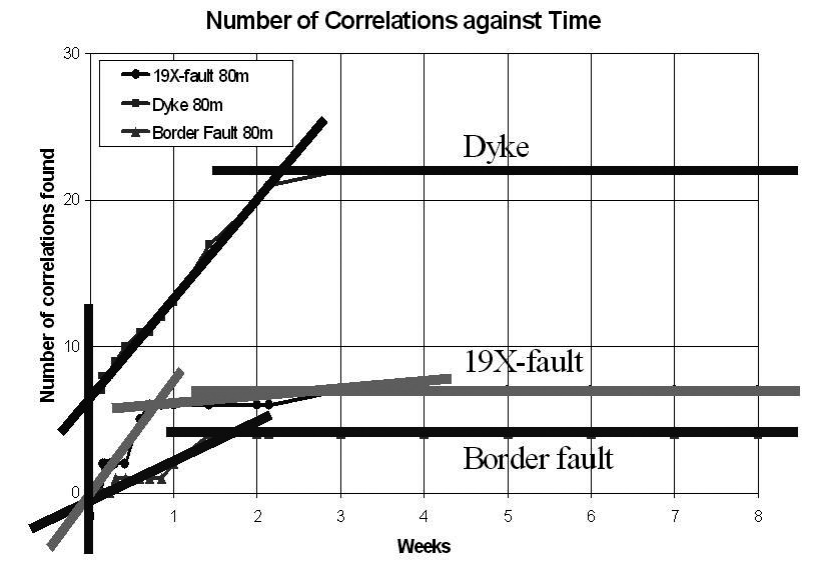

FIG. 14 Defined risk periods after the occurrence of a bigger event on a specific structure of interest

\section{CONCLUSIONS FROM TARGET SEISMIC DATA}

By using the time-space relationship between events the following was concluded:

- Event clusters can be readily identified.

- The migration of events on geological structures can be easily followed.

- Risk profiles for the whole mine as well as for individual geological structures can be obtained from the number of correlations against time.

- The chance for associated bigger events are greater shortly after the first event occurs and it then gradually decreases to the background value in 2-3 weeks.

- Clearly defined risk periods could be distinguished for all the geological structures investigated.

- The bigger events appear mostly uncorrelated with nearby small events in time, but appear to be better associated with other bigger events in the vicinity in time.

\section{ACKNOWLEDGMENTS}

The management of Harmony Target Mine for the use of the information obtained from the seismic network.

\section{REFERENCES}

Basson, F.R.P. (2002) Time dependant stope closure in the underground laboratory. South African National Institute of Rock Engineering 2002 Symposium (Re-defining the boundaries).

Basson, F.R.P. (2003) SeismicsInTime. Windows program used for seismic event correlation and available for free from fbasson@absamail.co.za.

Mendecki, A.J. (1997) Seismic monitoring in mines. Chapman and Hall, London.

Wiles, T. (2004) Map3D. Mine Modelling Pty Ltd (Website http:/ /www. map3d.com). 